\title{
Multimediale Kommunikation für Entwicklung in Tansania
}

\author{
von Josef Healy
}

"Während andere auf dem Mond landen, versuchen wir unsere Dörfer zu erreichen“, hat Julius Nyerere, der Präsident von Tansania, einmal gesagt. Seine Worte zeigen durchaus, um was es in der sozialen und ökonomischen Entwicklung vieler afrikanischer Länder geht. Die Dörfer zu erreichen bedeutet, den Leuten auf lokaler Ebene zu helfen, es bedeutet, mit ihnen auf einfachster Stufe in Kommunikation zu treten. Hier geht es mir darum, ein solches Kommunikationsprogramm für einfache Leute zu beschreiben, das im Maswa Distrikt, in der Shinyanga-Region West-Tansanias etfolgreich durchgeführt wurde ${ }^{1}$. Das Programm umfaßt den Einsatz verschiedener Kommunikationsmöglichkeiten von traditionellen einheimischen Formen über visuelle und audiovisuelle Mittel bis zur Presse. Es ging darum, mit dem koordinierten und konzertierten Gebrauch der Kommunikationsmittel einfache Frauen in den Dörfern zu erreichen. Das Experiment wird von der katholischen Kirche in Zusammenarbeit mit anderen christlichen Kirchen und staatlichen Stellen durchgeführt.

Leiter des Programms waren zwei amerikanische Missionsschwestern und zwei einheimische Frauen. Obwohl eine dieser Frauen nur eine Volksschulausbildung hatte, wurde sie von den Dorffrauen doch als "eine von uns" angenommen. Im Gegenteil, so meinten die Frauen des Dorfes, es sei besser so, denn wenn diese Frau zu einer besonderen Fachausbildung nach auswärts gegangen wäre, würde sie als „Expertin“ zurückkommen, die nicht mehr als „eine von uns" aufgenommen worden wäre. Dieses Vertrauen und die Glaubwürdigkeit sind ein entscheidender Punkt für erfolgreiche Kommunikation. Das Team der vier Frauen wird bei seiner Arbeit gelegentlich von weiteren einheimischen Schwestern und Laien unterstützt, die auch in dieser Region arbeiten.

Das Team der „Kommunikatoren“ führt eine Serie von fünftägigen Entwicklungsseminaren für Frauen der Shinyanga-Region durch. Die meisten dieser Kurse finden in den einheimischen Dörfern statt. Manchmal aber werden auch auf Wunsch der einheimischen Verwaltungsbeamten Kurse in der landwirtschaftlichen Ausbildungsstätte nahe der Stadt Shinyanga veranstaltet.

Die Teilnehmerinnen der Kurse sind jeweils 25 bis 30 Frauen aus den Dörfern des Maswa Distrikts. Man muß natürlich die Verhältnisse kennen, aus denen diese Frauen kommen und unter denen sie leben, um mit ihnen ins Gespräch zu kommen. Das Team machte dabei u. a. folgende Erfahrungen:

1. Die meisten dieser Frauen haben Kisukuma als ihre erste und Suaheli als ihre zweite Sprache. Die Kurse wurden aber in Suaheli gehalten, und alle Hilfsmittel sind in Suaheli abgefaßt. Dies geschieht bewußt, um Suaheli als die offizielle und nationale Sprache zu fördern und um ein Nationalbewußtsein zu entwickeln. Hinzu kommt, daß Lernhilfen in Suaheli in ganz Tansania und Kenya gebraucht werden können. 2. Die meisten dieser Frauen können nicht lesen. Das scheint den Gebrauch von gedruckten Hilfen auszuschließen. Tatsächlich fand man aber in den Familien dieses

P. Josef Healy mm, M. A. ist Sekretär für Kommunikation im Sekretariat der „Association of the Episcopal Conferences of Eastern Africa" (AMECEA) in Nairobi. Er ist in dieser Eigenschaft verantwortlich für die Kommunikationsprogamme der katholischen Kirche in Uganda, Kenya, Tansania, Malawi und Sambia. 
Gebietes zumeist wenigstens eine Person je Familie, die lesen kann. Deswegen waren einfache Blätter mit Illustrationen trotzdem erfolgreich. Auch Plakate mit Bildern und Zeichnungen waren sehr hilfreich.

3. Die Lehrmethoden und das Unterrichtsmaterial waren alle auf einheimische Dörfer abgestimmt. So wird während des Kursus der gleiche Typ von Kochtopf gebraucht, wie ihn die Frauen der Dörfer tagtäglich zuhause verwenden. Auf den Plakaten und in den Zeichnungen werden nur Gemüse und Früchte dargestellt, die überall im Garten oder auf dem Markt zu bekommen sind.

4. Noch eine weitere, sehr aufschlußreiche Erfahrung: Wenn die Frauen allein am Kurs teilnehmen und mit neuen Ideen und Methoden nad Haus zurückkehren, erfahren sie oft den Widerstand ihrer traditionsbewußten Männer. Deswegen werden auch die Männer eingeladen, entweder ganz oder doch teilweise am Kurs teilzunehmen, damit sie sehen, was ihre Frauen lernen. Es gibt ein eigenes Informationsblatt für die Männer.

Inhaltlich geht es bei den Kursen um bessere Methoden beim Kochen und Nähen, um Gesundheits- und Kinderfürsorge, Haushalt, Landwirtschaft und politische Bildung (vgl. Anhang).

\section{Publizistische Methoden}

Bei den fünftägigen Kursen geht es vor allem darum, durch die Praxis zu lernen. Jeder Unterrichtsstunde folgt eine praktische Übung, etwa: einen Kuchen backen, ein Kleid nähen, ein Baby baden. Um das zu erreichen, werden zunächst die traditionellen afrikanischen Kommunikationsformen gebraucht: Unterhaltung von Mensch zu Mensch, Gruppendiskussion (Palaver), Gruppenaktivität, Gesänge, Gesten und Signale, Drama und Theater. Diese Mittel und Methoden sind Grundlage des gesamten Kommunikationsgeschehens. Dorffrauen brauchen einen guten Teil persönlicher Ansprache und das Erlebnis des Dabeiseins durch gewöhnliche Unterhaltungen und Gruppenaktivitäten. Einige Lieder zur Hygiene, wie z. B. zum Fliegenfangen und Händewaschen vermitteln wichtige Vorstellungen für eine bessere Entwicklung.

Die Aufmerksamkeit der Frauen läßt sich nach den Erfahrungen des Unterrichtsteams am besten durch eine Fülle von Gesten und Bewegungen erhalten. Die Lehrerin mimt oder spielt die richtige und - im Gegensatz - die falsche Art und Weise, etwas zu tun. Das bringt die Frauen zum Lachen und das, was ihnen an neuen Ideen und Methoden beigebracht werden soll, kommt ohne Schwierigkeiten an. Dramatisierte Darstellungen werden sehr erfolgreich verwendet, wenn man die vorhergehende und nachfolgende Situation im Stand der Entwicklung zeigen möchte. So wird z. B. im ersten Teil ein schmutziges Haus dargestellt, während der zweite Teil ein sauberes, aufgeräumtes Haus zeigt.

Weiter werden visuelle Mittel zur Kommunikation gebraucht. So werden Plakate benutzt, die leicht vom Gesundheitsministerium in Dar-es-Salaam oder von der „Food and Nutrition Commission" aus Lusaka zu bekommen sind. Plakate mit unterernährten und wohlgenährten Kindern zeigen den Müttern den Unterschied. Die Bilder sind so lebensgetreu, daß sie schnell von der Bedeutung der Gesundheit für ihre Kinder überzeugt sind. Plakate für die drei Grundnahrungsmittelgruppen (Aufbau-, Energieund Schutzstoffe) benutzen entweder Fotos oder Zeichnungen. Wandkarten sind besonders wirksam beim Ernährungs- und Gesundheitsunterricht. Die Nahrungsmittelgruppen werden auf drei Schreibmaschinenbögen vervielfältigt und dann zu Nahrungsmittelkarten zusammengeklebt. Jede Frau hat während des Kurses diese 
Darstellungen der verschiedenen Nahrungsmittelkarten bunt zu malen und nimmt dann die fertige Karte mit nach Haus. In die ursprünglichen Falten gelegt, wird die Karte vor Knicken geschützt und ist leicht zu transportieren.

Klappkarten sind beim Unterricht für alphabetisierte Frauen hilfreich. Flanelltafeln helfen zur Besprechung der Grundnahrungsmittel. Die Bilder können von vorhandenen Fotos, Illustrationen und Zeichnungen, von Plakaten und anderen bestehenden Drucksachen genommen werden, oder man gebraucht bereits fertige Flanellbilder wie sie z. B. die All-Afrikanische Kirchenkonferenz herausgebracht hat. Die Gruppenbeteiligung der Frauen wird dadurch angespornt, daß sie die Bilder der verschiedenen Nahrungsmittel in die dazugehörigen Gruppen auf der Flanelltafel einordnen müssen. Anhand der Flanelltafel erzählt eine der Lehrerinnen auch die Geschichte einer modernen tansanischen Frau, die ihr Haus sauber hält, Gemüse im Garten pflanzt, damit ihre Familie Nahrung hat, die Hühner hält und ihren Kindern beibringt, im Haushalt zu helfen.

Während des Kurses werden Fotos gemacht, die dann bei den folgenden Seminaren und in anderen Dörfern gezeigt werden, damit die Frauen ihre Freundinnen als Kursteilnehmer entdecken können.

Gedruckte Unterlagen werden auf vielerlei Art und Weise eingesetzt. Ein vierseitiges Faltblatt unter dem Titel „Malezi ya Mama Nyumbani“ (Lehrstücke für die Mutter im Haus) war sehr erfolgreich. Das Blatt verwendet Fotos von Frauen aus Sukuma im Maswa Distrikt und beschreibt durch viele Illustrationen die Nahrungsmittelgruppen, gibt einheimische Rezepte und enthält insgesamt wenig, aber großgedruckten Text. Ursprünglich wurden 5000 Exemplare gedruckt. Sie wurden als Handzettel bei den Entwicklungskursen gebraucht und der vierteljährlich für die Victoria-See-Region West-Tansanias erscheinenden Zeitschrift "Tupeane Habari“ (Nachrichtenaustausch) beigelegt. Die Illustrationen des Handzettels wurden außerdem von drei Zeitungen nachgedruckt, von der evangelischen Zeitschrift "Lengo", dem katholischen „Kiongozi $^{\circ}$ und einer Regierungsveröffentlichung. Eine zweite Auflage wurde mit 4000 Exemplaren in Tansania und mit 2000 Exemplaren in Kenya durch das „Freedom from Hunger Council" für nationale Entwicklung in Kenya verteilt.

Eine Serie von 24 vierseitigen Blättern unter dem Titel "Malezi ya Nyumbani ${ }^{\alpha}$ (Unterricht für daheim) ist in Vorbereitung. Diese Blätter können später in einer Mappe zusammengelegt werden. Sie sollen in 5000 Exemplaren bei den Entwidklungskursen im Maswa Distrikt und in weiteren 5000 Exemplaren bei einem UNESCOorientierten Erwachsenenbildungsprogramm am Viktoriasee in Tansania verteilt werden. Die ersten fünf Blätter behandeln Themen wie Babynahrung, Nutzen einer Klinik, Toilettenhygiene, Bohnen als Nahrung. Ein Rezeptbuch unter dem Titel „Kitabu cha Uokaji“ ist bei den Frauen sehr beliebt. Es enthält 20 Rezepte mit Illustrationen auf jeder Seite. Sie sind schlicht geschrieben, so daß auch eine einfache Dorffrau leicht folgen kann.

Regeln für das Nähen werden mit Matrizen vervielfältigt und an die Frauen verteilt. Eine Serie von Zuschneidebögen ist außerdem als Beilage der Zeitschrift "Kiongozi“, die alle zwei Wochen mit 20000 Auflage erscheint, veröffentlicht worden.

Das Unterrichtsteam verteilt jeden zweiten Monat ein vervielfältigtes Informationsblatt „Habari za Akina Mama“, um den Müttern zu zeigen, was andere Frauen in ihren Dörfern tun und um die einheimischen Verantwortlichen über die Entwicklungsarbeit zu unterrichten Auch dieser Informationsdienst enthält Rezepte sowie Hinweise für Schneiderarbeiten. 
Ausstellungen mit den gefertigten Sachen am Ende des Kurses sind sehr beliebt. Jede Frau zeigt stolz ihre Näharbeiten, was sie sonst gemacht oder woran sie gearbeitet hat. Gewöhnlich kommen dann die Verantwortlichen des Dorfes oder der Gegend, um die Ergebnisse des Kurses zu sehen.

Eine dritte Gruppe von Kommunikationsmitteln sind die audiovisuellen Medien. Da werden Dias mit einem gesprochenen oder gesungenen Kommentar vorgeführt. Der aus dem Stegreif gegebene persönliche Kommentar spricht persönlich stärker an als Tonbanderläuterungen. Auch kann sich der Sprecher dabei besser an die jeweils gegebenen Umstände anpassen. Dias mit den Frauen selbst in ihrer einheimischen Umgebung sind besonders wirkungsvoll. So kann z. B. ein Fortbildungskurs mit einer Diavorführung über die täglichen Aktivitäten der vorhergehenden Kurse beginnen.

Kurzfilme über Ernährung, Hygiene und landwirtschaftliche Themen werden verhältnismäßig oft vorgeführt. Auch hier ist der Kommentar manchmal „live“, manchmal gesungen.

Der Super-8-mm-Film ist einer der interessantesten Teile des Programms. Vom Kursteam wurde mit Hilfe einheimischer Fachleute eine Serie von Super-8-mm-Filmen in Suaheli begonnen. Sie wurden im Maswa Dialekt aufgenommen und sind bei weitem wirkungsvoller als teure Filme aus anderen Teilen Tansanias oder Ostafrikas. Der erste Film "Safari Maendeleo“ (Entwicklungssafari) wurde im September 1971 vollendet. Er kostete nur 288,00 DM. Der Elfminutenfilm gibt eine Zusammenfassung eines typischen Fünftagekurses und behandelt Themen wie Nahrungsmittelgruppen, wie man ein Baby badet, wie man einen Topfkuchen backt, Kinderkleider macht, das Haus reinigt usw. Der Film wird zur Einführung bei bestimmten Kursen gebraucht oder als Rückschau bei anderen. Ein zweiter solcher 8-mm-Film über Kinderfürsorge wird gerade hergestellt.

16-mm-Filme werden oft vorgeführt. Zu ihnen gehören die Filme des Kinomobils der Regierung, das vom regionalen Informationsbeamten in Shinyanga geliehen wird. Hörfunk ist das letzte Medium in dieser multimedialen Kampagne auf lokaler Ebene. Das Team des Maswa Distrikts versucht seine Kurse mit den nationalen Bildungskampagnen des Instiutes für Erwachsenenbildung in Dar-es-Salaam zu koordinieren. Dies begann mit der "Mtu ni Afya" (Gesundheit ist Leben)-Aktion, die im November 1972 begonnen wurde. Hier gebrauchte man Radioprogramme über Gesundheit, gedrudkte Unterlagen und Studiengruppen. Die Frauen des Maswa Distrikts (vor allem solche aus den Ujamma Dörfern) wurden ermuntert, die Rundfunkprogramme in Verbindung mit den Gesundheits- und Hygienethemen des Fünftagekurses zu hören.

\section{Feedback und Beurteilung}

Mittelpunkt dieses multimedialen Kommunikationsversuchs ist die Betonung des koordinierten und konzentrierten Gebrauchs verschiedener Kommunikationsmittel, wie sie im Anhang I noch einmal übersichtlich dargestellt werden.

$Z$ wei grundsätzliche Fragen müssen hier gestellt werden:

1. Was ist die wirkungsvollste Strategie im Gebrauch der verschiedenen Kommunikationsmittel? Hier müßte beachtet werden:

a) die Häufigkeit, mit der die verschiedenen Mittel eingesetzt werden;

b) die Art und Weise (oder auch die Reihenfolge) der Verwendung;

c) die Integration der verschiedenen Mittel. 
2. Welche Beziehung haben die modernen Kommunikationsmittel (z. B. Film, Dias, Plakate, Flanelltafel, Gedrucktes) zu den traditionellen Mitteln afrikanischer Kommunikation (persönliches Gespräch, Gruppendiskussion, Gesang, dramatisierte Darstellungen) bei der Weitergabe von Ideen und Fertigkeiten im Bereich menschlicher und gesellschaftlicher Entwicklung?

Vorläufig läßt sich dazu aus den bisherigen Erfahrungen vielleicht folgendes sagen:

1. Der Gebrauch einer weiten Skala von Kommunikationsmitteln beeindruckt die Frauen des Maswa Distrikts während ihres Fünftagekurses sehr. Sie lieben die traditionellen afrikanischen Kommunikationsarten wie Gesang, Gruppendiskussion (Palaver) und Gruppenteilnahme. Sie reagieren positiv auf visuelle Kommunikationsmitel und sind oft von einer Dia-Vorführung und von Filmdarbietungen begeistert.

2. Dieses positive Feedback im Gebrauch vieler Kommunikationsmittel führt aber zu einer grundsätzlichen und wichtigen Frage: Verschnellert diese multimediale Methode tatsächlich den Lernprozeß und vor allem die Einführung neuer Ideen für den Entwicklungsprozeß? Nach eineinhalb Jahren Erfahrung in West-Tansania muß man hier mit Ja antworten. Auch wenn noch etliche Untersuchungen anzustellen sind, läßt sich vielleicht schon folgendes dazu sagen:

a) Je mehr menschliche Sinne am Kommunikationsprozeß teilnehmen, desto schneller lernen die Frauen neue Methoden. So ergab die gemeinsame Ansprache von Ohr, Auge und Hand zusammen die schnellsten und besten Ergebnisse.

b) Die verschiedenen Medien haben einen großen Verstärkungseffekt (Reinforcement). So ist der 8-mm-Film bei einem typischen Fünftagekurs eine ausgezeichnete Einführung am ersten Tag eines neuen Kurses, aber auch eine gute Zusammenfassung am letzten Tag.

c) Visuelle Hilfen, die die Frauen während des Kurses selbst herstellen, und mit nach Hause nahmen (z. B. die Nahrungsmittelkarten) haben eine große Nachwirkung ("Follow up").

d) Gedruckte Hilfsmittel empfinden die Frauen als sehr hilfreich. Eine positive Reaktion auf Fotos, die an Ort und Stelle gemacht wurden, sowie viele sorgfältig geschnittene einfache Illustrationen, wenig Text in großem Druck vertiefen die Ergebnisse anderer Erwachsenenbildungsprogramme.

e) Die herzliche Aufnahme des Unterrichtsteams als Menschen, die persönlich am Leben der Frauen interessiert sind, und die einheimische Umgebung halfen, die multimediale Ansprache wesentlich wirksamer zu machen.

Selbstverständlich sind noch weitere Untersuchungen und Studien notwendig. Hier sollte eine erste Übersicht versucht werden.

\section{A NHA NG I}

Koordinierter Gebrauch verschiedener Kommunikationsmittel

Kommunikationsmittel :

1. persönliches Gespräch

2. Gruppendiskussion gebraucht für:

alle Kurse

alle Kurse, speziell Kochen,

Gesundheitspflege, Kinderpflege
Häufigkeit:

oft

of $t$ 
Kommunikationsmittel:

3. Gruppenaktivitäten

4. Gesang

5. Gesten und Signale

6. Drama(theater)

7. Plakate

8. Wandkarten

9. Klappkarten

10. Flanelltafeln

11. Ausstellung von Bildern

12. Gedruckte Unterlagen - Handzettel, Mitteilungsblätter, Beilagen zu Zeitschriften, usw.

13. Ausstellung von Arbeiten

14. Dias - mit ,live' Kommentar oder gesungenem Kommentar

15. Kurzfilme - mit ,live oder gesungenem Kommentar

16. Super $8 \mathrm{~mm}$ Film

17. $16 \mathrm{~mm}$ Film

18. Hörfunk (verbunden mit nationalen Programmen wie jenem des Instituts für Erwachsenenbildung) gebraucht für:

Häufigkeit:

Kochen, Gartenarbeit, Haus- of arbeit

Kochen, Gesundheitspflege, manchmal polit. Bildung, Auswendiglernen von Rezepten und

Regeln für die Gesundheitspflege

alle Kurse - besonders bei ziemlich of

Nähen und Handarbeit

polit. Bildung, Hygiene

Ernährung, Kochen, Hygiene,

Politik, Kinderpflege

Ernährung, Kochen, Gesund- oft

heitspflege, Kinderpflege

Hilfe in Seminaren für alpha- manchmal

betisierte Frauen

Gesundheitspflege, Hausarbeit

Nähen, Kochen

a) Kochen (Rezeptbuch)

b) Nähen

c) Handzettel über Ernährung, Gesundheits- und

Hauspflege

d) Nachrichtenblatt über

Entwicklungsaktivitäten und praktische Vorschläge

Nähen und Handarbeit

alle Themenbereiche

alle Themenbereiche

ziemlich of

ziemlich oft oft

ziemlich of ziemlich of $t$

ziemlich of $t$

alle Themenbereiche

Gesundheitspflege, Landwirtschaft, Kinderpflege, Politik

Gesundheit, Landwirtschaft,

Politische Bildung ziemlich of ziemlich oft

manchmal

\section{A NHANG I I}

Inhalt des Entwicklungsprogramms (Auszug)

I. Der Unterricht im Kochen beinhaltet:

1. Diskussion über die zu kochenden Nahrungsmittel und deren Wert (Gebraudh der Nahrungsmittelkarte).

2. Zeigen der einzelnen Zutaten, eine nach der anderen und Vorführung, wie man sie abmißt oder wieviel davon gebraucht wird (Gebrauch des Rezeptzettels oder Vorlesen oder Vorsingen der Rezepte durch eine Frau). 
3. Vorführung der Vorbereitung des Vermischens und Kochens oder Backens (Mittelbare Teilnahme - Hilfe verschiedener teilnehmender Frauen).

4. Diskussion der Ergebnisse

5. Ausmustern der Nahrungsmittel

6. Unmittelbare Teilnahme am folgenden Tag: Frauen in Gruppen zu drei oder vier bringen die entsprechenden Nahrungsmittel von daheim mit und kochen jetzt selbst unter Aufsicht der Lehrerin.

7. Diskussion der Ergebnisse: Durchsicht des Rezeptes, vielleicht in Form eines einprägsamen Rezeptsongs.

8. Nacharbeit der Lehrerin: Die Dörfer besuchen, um die Frauen zu sehen und mit ihnen ihre Schwierigkeiten zu besprechen, und zu sehen, wie sie sich entwickeln.

9. Veröffentlichung des Rezeptes in einem Handzettel, in einem kleinen, alle zwei Monate erscheinenden Nachrichtenblatt. Verkauf von Rezeptbüchern an die Frauen.

10. Vorführung der Fotos der kochenden Frauen.

II. Der Unterricht im Nähen umfaßt:

1. Auslegen jener Kleidungsstücke und Gegenstände, die zum Unterricht nötig sind.

2. Diskussion über den Bedarf der Frauen und der Preise für die Zutaten und Stoffe, um den Frauen bei ihrer Wahl zu helfen.

3. Persönliche Vorführung des Zuschneidens, Nähens, Vorzeichnens der Stickerei, Perlarbeit usw. Dies kann auch in größeren Gruppen getan werden, wenn vorher einige Frauen in der Handarbeit vorgebildet sind - oder wenn einige eher fertig sind als andere.

4. Ständige Aufsicht und Ermunterung von seiten der Lehrerinnen um Fehler und Entmutigungen zu vermeiden.

5. Ausstellung von den Frauen fertiggestellter Artikel an einem Aushängebrett oder auf Tischen. Sobald sie fertig sind, ihre Arbeiten anderen zeigen.

6. Gruppenfotos der Frauen bei der Arbeit.

7. Stickereizeichen oder Zuschneideblätter und Nähideen können auch im vervielfältigten Nachrichtenblatt oder auch in nationalen Zeitschriften wie "Kiongozi" und "Lengo" erscheinen.

III. Unterricht in Gesundheitspflege, Kinderpflege und Hausarbeit:

1. Diskussion eines Problems (z. B. Malaria) oder einer Fertigkeit (z. B. Baden eines Babys), um festzustellen, was die Frauen davon wissen.

2. Einfache Darstellung durch Flanelltafel, Plakate oder Karten, Dias oder durch tatsächliches Tun.

3. Diskussion und Fragen

4. Gruppenaktivitäten wie z.B. ein Moskitonetz machen, den Hof säubern, Baden einiger Babys durch einige Frauen und nachher Kritik ihres Versuchs durch die Gruppe; den Frauen Saatgut geben zum Säen daheim - oder Austausch von Saat.

5. Verteilung von Handzetteln zum Thema nach dem Unterricht, z. B. „Malezi ya Mama Nyumbani" (Unterricht für die Mutter in ihrem Haus).

6. Zeigen von Fotos anderer Frauen in ähnlicher Situation; Fotografieren der arbeitenden Frauen, um die Fotos später zu zeigen.

7. Besuche in den Dörfern nachher, um zu sehen, wie die neuen Kenntnisse angewandt werden.

Anmerkung:

1. Vgl. auch den Beitrag des gleichen Verfassers über „Kommunikationsausbildung in Tansania" in CS 4:1971, S. 233-235. Ferner den Bericht von Theobald Kyambo und N.E. P. Mtowe in diesem Heft S. 53-57. 


\section{S U M M A R Y}

A team consisting of two Sisters and two laywomen has, for almost two years, been conducting Development Courses which last about five days, in the Maswa District of the Shinyanga Region in West Tanzania. They teach the local women cooking, nutrition and hygiene, childcare, needlework, civic education and agriculture. The courses are usually held in the villages. A wide range of communications media is used. Local African forms of communication, such as person-to-person conversations, group discussion, group activities are also used with songs, gestures and signs, drama posters, wall charts, flip charts, flannelgraph pictures, displays, printed materials, slides, film-strips, super 8 and $16 \mathrm{~mm}$ films and radio. The more all the senses are brought into the learning process, the quicker the women are to acquire new skill. Modern media, especially film, has a great reinforcement value. Visual aids which can be made by the women during the course and taken home by them have great follow-up value. Great importance is attached to the fact that the women in the villages have confidence in the team.

\section{RESUME}

Dans le district de Maswa, dans la région de Shinyanga, en Tanzanie Occidentale, une équipe d'enseignantes organisent depuis un an et demi des cours pour les femmes indigènes; ces cours durent cinq jours et touchent à tous les domaines de la vie quotidienne (cuisine, alimentation, puériculture, hygiène, couture, formation politique et agricole). Ils ont lieu dans la mesure du possible dans les villages. On y utilise un vaste éventail de moyens journalistiques qui vont des formes africaines locales telles que le dialogue personnel, les discussions et les activités de groupe ayant pour objet chants, mimes, signaux, représentations dramatiques, affiches, cartes murales, expositions, documents écrits, diapositives, courts métrages, films $8 \mathrm{~mm}$ et $16 \mathrm{~mm}$ jusqu'à la radio qu'on introduisit dans les cours dans le cadre des campagnes nationales. Plus le nombre de sens mis en jeu pour l'enseignement est grand, plus les femmes acquièrent rapidement de nouvelles méthodes. Les moyens de communication de masse modernes, plus spécialement le film, ont contribué à un plus grand approfondissement; les aides visuelles, à la confection desquelles les femmes elles-mêmes participèrent et qu'elles peuvent ensuite emporter chez elles, influent particulièrement sur l'assimilation des cours auxquels elles ont pris part. Il ne faut pas non négliger le fait que l'équipe d'enseignants possède l'entière confiance des femmes indigènes.

\section{R E S UMEN}

Desde hace año y medio un equipo de cuatro maestras dan cursos intensivos de cinco días de duración en el districto de Maswa, en la región tanzanesa occidental de Shinyanga, destinados a formar a las mujeres de la zona en todos los estadios de la vida cotidiana (cocina, alimentación, cuidados sanitarios, puericultura, higiene, costura, educación politica y agricola). Estos cursos se organizan, en la medida de lo posible, en las mismas aldeas. Para ello se hace uso de un amplio conjunto de medios propagandísticos que son incorporados a los cursos en el marco de campañas nacionales y que abarcan desde modalidades características africanas, como la conversación personal, la discursión en grupo, las actividades conjuntas como canto, mimo, señales, representaciones dramáticas, pancartas, murales, manufacturados de franela, exposiciones e impresos, hasta diapositivas, películas cortas y películas de 8 y 16 milímetros e incluso la radio. Cuanto mejor se coordina el empleo de estos medios en el proceso didáctico, con mayor rapidez aprenden las alumnas nuevas habilidades. Los modernos medios de comunicación, sobre todo el cine, tienen además un considerable efecto para la retentiva; los recursos visuales, cuando han sido preparados por mujeres autóctonas en proceso de aprendizaje, tienen una mayor eficacia para las que después acuden a los cursos. También es importante que el equipo de maestras goce de la plena confianza de las mujeres autóctonas. 